\title{
Properties of MR Transmission under Thermal Affect
}

\author{
Jin Huang ${ }^{*}$, Zhen Qiao and Beibei Fan \\ Chongqing Automobile College, Chongqing University of Technology, Chongqing, China \\ *Corresponding author
}

\begin{abstract}
A transmission method of magnetorheological(MR) fluid in disc type driven by shape memory alloy (SMA) spring with the thermal effect was proposed. The torque changed at difference temperatures. Based on the thermal effect of SMA, the expression for displacement of SMA spring was established and the rheological properties of MR fluids under the magnetic fields was described by Herschel-Bulkley model. The shear flow of MR fluid between two discs was analyzed by the momentum equation and the expressions of flow velocity and torque were obtained. The experimental results show the displacement of SMA spring and the transmit torque of MR Fluid are directly proportional to the temperature and the applied magnetic field, respectively. The transmit torque of MR Fluid can be continuously controlled by the SMA spring in difference temperatures.
\end{abstract}

Keywords-thermal affect; SMA spring; MR fluid; transmission property

\section{INTRODUCTION}

Shape memory alloys (SMA), according to their property to recover a prefixed shape every time heated above the transformation temperature. The characteristics of restoring force for SMA can be employed both as sensor or actuator [12]. This kind of component is known as simple structure, high sensitivity and good stability. The SMA formed actuator can be applied to wind direction adjusting mechanism for an air conditioner, Micropump, Microvalve, thermostatic mixing valve, fire resistant valve for gas pipeline, sprinkler fire extinguishing system, robot hand derived by shape memory alloy actuator, auto thermostat, automobile fan clutch and automatic actuator for fog lamp cover etc. [3-4].

Magnetorheological fluid (MRF), is magnetic polarization of solid micro particles in the liquid formed the suspension [5], its rheological properties can be made of applied magnetic field control continuously. Without the applied magnetic field, MRF showed similar Newtonian fluid behavior [6]. In the applied magnetic field, MRF showed yield stress obviously, the yield stress can be made of applied magnetic field intensity continuous control [7]. The magnetorheological technology has potential applications in many industries. Based on the structure, the magnetorheological technology can be applied in the next generation product, the power density, precision and dynamic performance as the main function characteristics of the product design [8-10]. The characteristics of MRF applied to transmission component are: it can realized wrapping connector driving between driving member and follower. Can realized high sensitivity, high precision and smooth stepless speed regulation. Easy to realized the process of speed control and automatic control. Changing driving shaft steering, its transmission properties do not change.
According to the transmission mechanism of MRF, MRF transmission can be divided into shear transmission, pressure transmission and extrusion transmission. The shear transmission of MRF is the use of MRF by the applied magnetic field control of the shear yield stress to provide transfer torque. Huang et al. analyzed of the transmission mechanism of MRF in the brake, established of a torque equation for cylindrical MRF brake, proposed the calculation method of MRF effective thickness in the brake [11]. Doruk Senkal et al. designed the magnetic circuit of MRF brake into serpentine, made the same size of the brake torque increase greatly [12]. Tran Hai Nam et al. introduced a new method to design MRF brake, made the the braking torque twice as high than conventional brake [13]. Kerem Karakoc et al. analyzed the design methods for MRF automobile brake [14]. V K Sukhwani et al. researched the method of design, development and performance evaluation for high speed MRF brake [15].

This paper used the SMA with temperature and driving characteristics and yield stress of MRF with applied magnetic field change characteristics, applied them to transmission installation. Made the MRF stored in oil chamber without work, which is beneficial to maintain the stability of MRF material performance, and improve the service life of MRF. SMA made sliding type temperature control switch used to control the excitation coil current of MRF, also helps to simplify the control system of magnetic rheological device, realized the transmission from the dynamic temperature and control, and the research results in automobile engine fan clutch, nuclear power plant centrifugal fan cooling systems, etc. Has the broad application prospect.

\section{TRANSMISSION PRINCIPLE}

The transmission installation of MR fluids driven by SMA spring with the thermal effect is shown in Figure. 1. When the temperature is lower than a setting temperature (e.g. $75^{\circ} \mathrm{C}$ ), the temperature control switch of SMA is in the off-state. No current is applied to the coils and the MR fluids are stored in oil chamber. There is no transmission medium in working gap and no torques can be the transmitted by installation. When the temperature is higher than $75^{\circ} \mathrm{C}$, SMA spring pushes MR fluids from oil chamber to working chamber and the coil current was produced by SMA control switch. The suspension particles in MR fluids are polarized and the chain structures formed by the magnetic field. The driving-disc and driven-disc are joined and the torques can be transmitted. The current is controlled by the temperature control switch of SMA responses to real-time temperature and torque can be adjusted by the temperature. When the temperature is less than $75^{\circ} \mathrm{C}$, SMA spring is compressed. The MR fluids flows back to oil 
chamber because of the centrifugal force and compressed air in working chamber. Then, driven-disc is stopped.

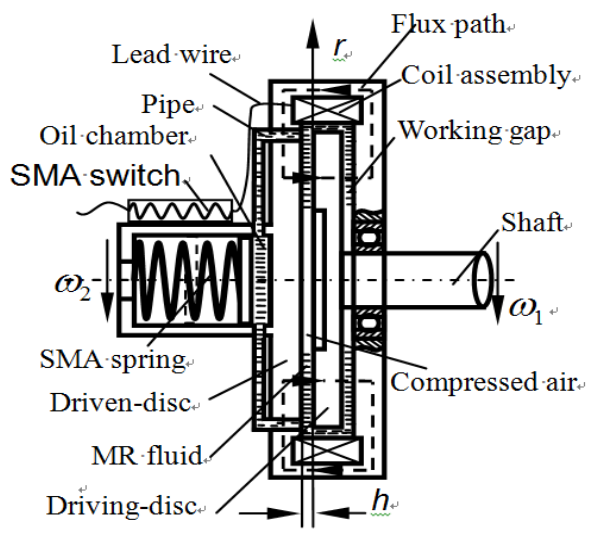

FIGURE I. THE PRINCIPLE OF MR FLUIDS TRANSMISSION IN DISC TYPE DRIVEN BY SMA SPRING

\section{THE SHEAR MODE OF MR FLUID BETWEEN TWO DISCS}

The shear model of MR fluid between two discs is shown in Figure. 2. The MR fluids work in the gap between two discs which has the inside radius, $R_{1}$, the external radius, $R_{2}$, and the distance of two disc, $h\left(h<<R_{2}-R_{1}\right)$. When the driving-disc rotates around the $\mathrm{z}$ direction with angular velocity, $\omega_{1}$, the driven-disc will rotate with angular velocity, $\omega_{2}$, by the shear transmission of MR fluids between two discs.

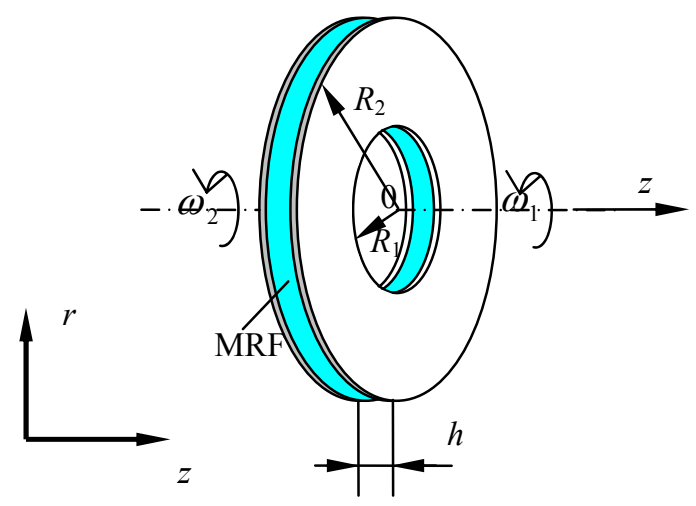

FIGURE II. SHEAR MODE OF MR FLUIDS BETWEEN TWO DISCS

In Figure. 2, the MR fluids are supposed to be incompressible fluids and symmetrical steady state flows $\left(\frac{\partial}{\partial \theta}=0, \frac{\partial}{\partial t}=0\right)$, the gravity is ignored, the MR fluids just have tangential flow velocity $\left({ }^{u_{\theta}}\right)$, and have no flow velocities along the radial and axial. The tangential flow velocity $\left(u_{\theta}\right)$ of MR fluid is a function of $z$, i.e, $u_{\theta}=u(z)$. According to the assumption conditions, the $\partial u_{\theta} / \partial \theta \equiv 0$, in other words, flow velocity component along the $\theta_{\text {direction is constant. The flow }}$ velocities along each coordinate direction are:

$$
u_{r}=0, u_{\theta}=r \omega(z), u_{z}=0
$$

where $r$ is the radius of circle, and $\omega(z)$ is the angular velocity of MR fluid along the direction of disc thickness.

In the cylindrical coordinate system, the motion equation of MR fluids along the $\theta$ direction between two discs is:

$$
\frac{\partial \tau_{z \theta}}{\partial z}=0
$$

where $\tau_{z \theta}$ is the shear stress of MR fluids.

From Eq. (2), we can obtain:

$$
\tau_{z \theta}=c_{1}
$$

where ${ }^{C_{1}}$ is the constant of integration.

As shown in Eq. (3), the shear stress is a constant in everywhere between two parallel discs when the MR fluid is in shear mode. If the MR fluids in somewhere are yielded, all of MR fluids in working gap are yielded. On the contrary, if somewhere is not yielded, all of fluids are not yielded.

When $\tau_{z \theta}<\tau_{y}$, the MR fluids in working gap are not yielded and similar to solid. The angular velocity of MR fluids in working gap is same in everywhere, and the angular velocity of input equal to the one of output.

$$
\omega(z)=\omega_{1}=\omega_{2}
$$

When $\tau_{z \theta}>\tau_{y}$, the MR fluids in working gap are yielded and in shear mode:

$$
\frac{\mathrm{d} \omega(z)}{\mathrm{d} z}=\frac{1}{r}\left(\frac{c_{1}-\tau_{y}(H)}{\phi(T)}\right)^{\frac{1}{m}}
$$

Applied the boundary conditions: $z=0, \omega(z)=\omega_{2}$; $z=h, \omega(z)=\omega_{1}$ to Eq. (5), the equation of angular velocity can be expressed:

$$
\omega(z)=\omega_{2}+\frac{\left(\omega_{1}-\omega_{2}\right) z}{h}
$$




\section{TRANSMISSION TORQUE}

The behavior of MR fluids is similar to Newtonian fluid without applied magnetic field. The constitutive equation can be described as [17]:

$$
\tau=\phi(T) \dot{\gamma}_{2}
$$

where $\tau$ is the shear stress of MR fluids, $\phi(T)$ is the viscosity of MR fluids $\dot{\gamma}_{2}$ is the shear strain rate of MR fluids.

When an magnetic field is applied, the characteristics of MR fluids can be described by Herschel-Bulkley model [18]:

$$
\begin{cases}\tau=\tau_{y}(H)+\phi(T) \dot{\gamma}_{2}^{m} & \tau \geq \tau_{y}(H) \\ \dot{\gamma}_{2}=0 & \tau<\tau_{y}(H)\end{cases}
$$

where $\tau_{y}(H)$ is the dynamic yield stress and $m$ is constant.

When the shear strain rate is $\dot{\gamma}=10000 \mathrm{~s}^{-1}$, the shear stress are $0 \mathrm{kPa}, 7.83 \mathrm{kPa}$, and $16.24 \mathrm{kPa}$ corresponded to the magnetic field strength $H$ are $0 \mathrm{kAmp} / \mathrm{m}, 90 \mathrm{kAmp} / \mathrm{m}$, and $185 \mathrm{kAmp} / \mathrm{m}$, respectively. When magnetic field is in stabile state(e.g. $H=$ $90 \mathrm{kAmp} / \mathrm{m}$ ), the shear stress are $12.32 \mathrm{kPa}, 14.13 \mathrm{kPa}$, $15.02 \mathrm{kPa}$ corresponded to the shear strain rate are $10000 \mathrm{~s}^{-1}$, $20000 \mathrm{~s}^{-1}$, and $25000 \mathrm{~s}^{-1}$, respectively. The results show that the shear stress is heavily influenced by applied magnetic field and is not obviously influenced by shear strain rate.

As shown in Figure. 2, MR fluids flow between two discs. The working area of disc is a ring which has radius from $R_{1}$ to $R_{2}$. A micro-ring in the radius $r$ was defined as $\mathrm{d} A$, and $\mathrm{d} A=2 \pi r \mathrm{~d} r$. The force produced by micro-ring was defined as $\mathrm{d} F$, and $\mathrm{d} F=\tau \mathrm{d} A$, where $\tau$ is the shear stress of MR fluids. The transmission torque is $\mathrm{d} M$, then, $\mathrm{d} M=r \mathrm{~d} F$, i.e, $\mathrm{d} M=2 \pi r^{2} \tau \mathrm{d} r$. The transmission torque of whole disc can be expressed:

$$
M=\int_{R_{1}}^{R_{2}} 2 \pi r^{2} \tau \mathrm{d} r
$$

There are two parts of transmission torque of MR fluids between two parallel discs, which are torque $M_{\eta}$ produced by viscosity of MR fluids and torque $M_{H}$ produced by yield stress $\tau_{y}$. If the all of MR fluids in working gap between two discs are yielded and in shear mode, the transmission torque $M_{\eta}$ produced by viscosity of MR fluids can be derived by combining Eqs. (6) , (7) and (9):

$$
M_{\eta}=\int_{R_{1}}^{R_{2}} d M_{\eta}=2 \pi \int_{R_{1}}^{R_{2}} \tau_{\eta} r^{2} d r=\frac{2 \pi \phi(T)}{m+3}\left(R_{2}^{m+3}-R_{1}^{m+3}\right)\left(\frac{\omega_{1}-\omega_{2}}{h}\right)^{m}
$$

The torque MH produced by yield stress of MR effect, $\tau_{y}(H)$ , can be described as,

$$
M_{H}=\int_{R_{1}}^{R_{2}} d M_{H}=2 \pi \int_{R_{1}}^{R_{2}} \tau_{y}(H) r^{2} d r=\frac{2 \pi}{3}\left(R_{2}^{3}-R_{1}^{3}\right) \tau_{y}(H)
$$

The transmission torque of MR fluids between two discs is

$$
\begin{aligned}
& M=M_{H}+M_{\eta} \\
& =\frac{2 \pi}{3}\left(R_{2}^{3}-R_{1}^{3}\right) \tau_{y}+\frac{2 \pi \kappa}{m+3}\left(R_{2}^{m+3}-R_{1}^{m+3}\right)\left(\frac{\omega_{1}-\omega_{2}}{h}\right)^{m}
\end{aligned}
$$

The inside and outside radius of working gap for MR fluid are assumed as $R_{1}=25 \mathrm{~mm}$ and $R_{2}=50 \mathrm{~mm}$, respectively. The working gap and the maximum input angular velocity are assumed $R_{2}=50 \mathrm{~mm}$ and $\omega_{1 \max }=100 \mathrm{rad} / \mathrm{s}$, respectively. By the experimental results, the index $m$ and the viscosity $\phi(T)$ can be obtained:

$$
\begin{aligned}
& m=0.93 \\
& \phi=143 \mathrm{e}^{-0.0143 T}
\end{aligned}
$$

From Eq.(10) we can calculate the maximum viscosity for transmission torque of MR fluid between two discs in different temperature, $\mathrm{T}$, without magnetic field. The theoretical results are compared with experimental results, which shown in Figure. 3. When the temperature is $20^{\circ} \mathrm{C}, 40^{\circ} \mathrm{C}, 80^{\circ} \mathrm{C}$, and $120^{\circ} \mathrm{C}$, the transmission torques of maximum viscosity is 1.11 N.m, 0.84 N.m, 0.47 N.m, and 0.27 N.m, respectively. The theoretical results are satisfied with the experimental results. The transmission torque of MR fluids has a great influence by temperature, and it will rapidly fall down with the increasing of temperature.

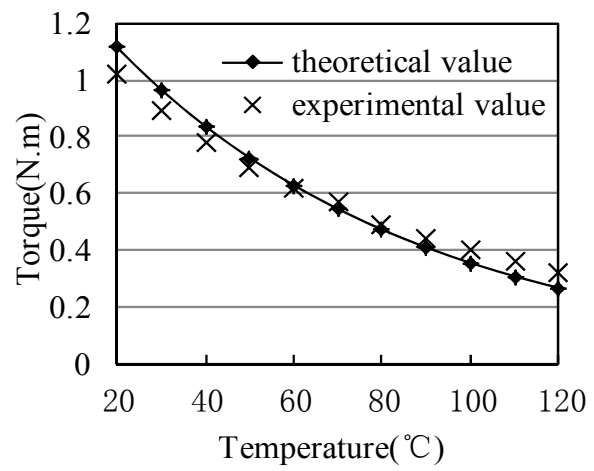

FIGURE III. TORQUE VS. TEMPERATURE WITHOUT MAGNETIC FIELD

When temperature is $40^{\circ} \mathrm{C}$, the relationship between the transmission torque and the magnetic field strength of MR fluids between two parallel discs was obtained according to Eq (12). The comparison of the theoretical and the experimental results are shown in Figure.4. When magnetic field strength is $40 \mathrm{kAmp} / \mathrm{m}, 80 \mathrm{kAmp} / \mathrm{m}$, and $160 \mathrm{kAmp} / \mathrm{m}$, the calculation results of transmit torque are 3.45 N.m, 5.74 N.m, and 9.22 N.m, respectively. When the saturation magnetic field strength 
is $220 \mathrm{kAmp} / \mathrm{m}$, the transmission torque is $10.49 \mathrm{~N} . \mathrm{m}$. The transmission torque of MR fluids is quickly enlarged until saturation with increasing of the magnetic field strength.

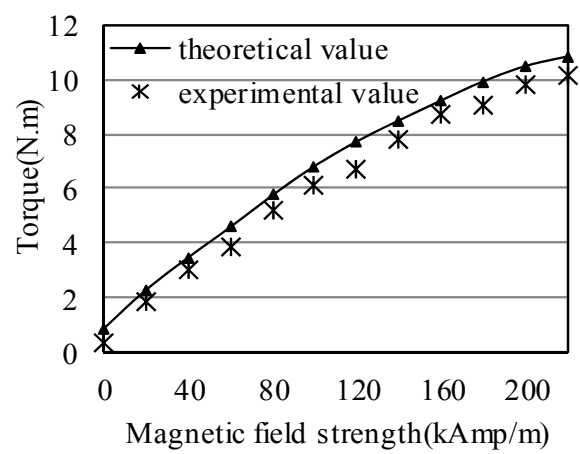

\section{FIGURE IV. TORQUE VS. MAGNETIC FIELD STRENGTH}

The SMA switch outputs $1 \mathrm{~mm}$, the driven current in the coil will increases $0.1 \mathrm{~A}$, and the magnetic field strength will increases about $10 \mathrm{kAmp} / \mathrm{m}$. The theoretical values and the experimental results of transmission torque influenced by temperatures are shown in Figure. 5. The figure presents that the transmission torque is directly proportional to the temperature act on SMA switch.

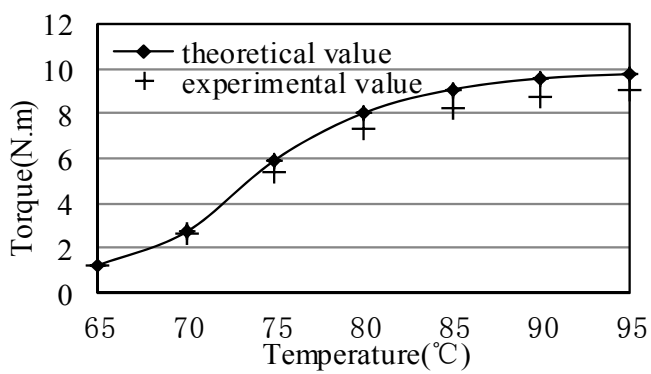

FIGURE V. TORQUE VERSUS TEMPERATURE WITH MAGNETIC FIELD

\section{CONCLUSIONS}

(1). Based on the heating and driving characteristics of SMA, the relationship between displacement and temperature of SMA Spring were analyzed. The expression of displacement for SMA Spring was established. The experimental results were well satisfied with the theoretical results. In phase transition temperature range, the displacement of SMA spring enlarged with the increasing of temperature.

(2). Based on rheological characteristics of MR fluids, the flow and transmission of MR fluids between two discs were analyzed, and the equations of flow velocity and transmission torque of MR fluids between two discs were obtained. MR fluids flow between two discs can be approximated to laminar flow, the transmission torque increased with the applied magnetic field.

(3). The MR fluids were driven to work chamber by SMA Spring. In the same time, the electric current was also adjusted by temperature. The transmission torque of MR fluids can be consecutively controlled and the value of it increased with the temperature.

\section{ACKNOWLEDGMENTS}

This work was supported by the National Natural Science Foundation of China $(51175532,11272368)$.

\section{REFERENCES}

[1] X.B. Tan, J. S. Baras and P.S. Krishnaprasad, "Control of hysteresis in smart actuators with application to micro-positioning," Syst. Cont. Lett., vol. 54, pp. 483-492, 2005.

[2] G. Costanza, M.E. Tata and C. Calisti, "Nitinol one-way shape memory springs: Thermomechanical characterization and actuator design," Sens. Actuators, A. Phys., vol. 157, pp. 113-117, 2010.

[3] S. Degeratu, P. Rotaru, G. Manolea, H. O. Manolea and A. Rotaru, "Thermal characteristics of Ni-Ti SMA (shape memory alloy) actuators," J. Therm. Anal. Calorim., vol.97, pp.695-700, 2009.

[4] S. Dilthey and H. Meier, "Simulation-Based Design of a Rotatory SMA Drive,” J. Mater. Eng. Perf., vol. 18, pp.686-690, 2009.

[5] G.L. Gulley and R. Tao, "Structures of a Magnetorheological Fluid," Int. J. Mod. Phys. B., vol.15, pp. 851-858, 2001.

[6] Y. Grasselli, G. Bossis and E. Lemaire, "Structure induced in suspensions by a magnetic field," J. Phys. II France. vol.4, pp. 253-263, 1994.

[7] S. K. Hyun, P. B. Jun and C. H. Jin, "Effect of magnetic nanoparticle additive on characteristics of magnetorheological fluid," IEEE Trans. Magn., vol. 45, pp. 4045-4048, 2009.

[8] J.D. Carlson, D.M. Catanzarite, and K.A. St. Claire, "Commercial Magneto-Rheological Fluid Devices,” Int. J. Mod. Phys. B. vol.10, pp.20-28, 1996.

[9] J. Huang, J.M. He and J.Q. Zhang, "Viscoplastic Flow of the MR Fluid in a Cylindrical Valve,” Key Eng. mater. vol.274-276, pp.969-974, 2004.

[10] J. Huang, J. M. He and G. P. Lu, "Analysis and Design of Magnetorheological Damper,” Adv. Mater. Res., vol. 148-149, pp. 882-886, 2011.

[11] J. Huang, J. Q. Zhang and Y. Yang, "Analysis and design of a cylindrical magneto-rheological fluid brake,” J. Mater. Proc. Technol, vol. 129, pp. 559-562, 2002.

[12] D. Senkal and H. Gurocak, "Serpentine flux path for high torque MRF brakes in haptics applications," Mechatronics, vol.20, pp.377-383, 2010.

[13] T. H. Nam and K. K. Ahn, "New approach to designing an MR brake using a small steel roller and MR fluid," J. Mech. Sci. Technol, vol. 23, pp.1911-1923, 2009.

[14] K. Karakoc, E. J. Park and A. Suleman, "Design considerations for an automotive magnetorheological brake," Mechatronics, vol. 18, pp. 434447, 2008.

[15] V. K. Sukhwani and H. Hirani, "Design, development, and performance evaluation of high-speed magnetorheological brakes," J. Mate. Desi. Appl. ,vol. 222, pp. 73-82, 2008.

[16] J.Z. Ma, H.Y. Shu, and J. Huang, "MR Continuously Variable Transmission Driven by SMA for Centrifugal Fan in Nuclear Power Plant," Sci. Technol. Nucl. Install., vol. 2012, pp. 1-6, 2012.

[17] J. D. Carlson and M. R. Jolly, "MR fluid, foam and elastomer devices", Mechatronics. vol.10, pp. 555-569, 2000.

[18] W. H. Herschel and R. Bulkley, "Konsistenzmessungen von GummiBenzol-Losungen”. Kolloid-Zeitschrift. vol. 39, pp. 291-300, 1926. 